Archives de sciences sociales des religions

147 | juillet-septembre 2009

Traduire l'intraduisible

\title{
Les fils du texte: Genèse 6, 1-4
}

\section{Marc de LAUNAY}

\section{CpenEdition}

Journals

Édition électronique

URL : http://journals.openedition.org/assr/21348

DOI : $10.4000 /$ assr. 21348

ISSN : $1777-5825$

Éditeur

Éditions de l'EHESS

Édition imprimée

Date de publication : 1 octobre 2009

Pagination : 41-59

ISBN : 978-2-7132-2217-7

ISSN : 0335-5985

Référence électronique

Marc de LAUNAY, "Les fils du texte: Genèse 6, 1-4 », Archives de sciences sociales des religions [En ligne], 147| juillet-septembre 2009, mis en ligne le 01 octobre 2012, consulté le 20 avril 2019. URL : http://journals.openedition.org/assr/21348 ; DOI : 10.4000/assr.21348 


\section{Marc de Launay}

\section{Les fils du texte : Genèse 6, 1-4}

Valéry rapporte ce mot de Talleyrand répondant à la question de savoir s'il croyait à la Bible: "J'ai deux raisons invincibles d'y croire, et d'abord parce que je suis évêque d'Autun; et ensuite parce que je n'y comprends absolument rien. " ${ }^{1}$ À travers ce trait d'esprit étayé d'un paradoxe, Talleyrand semble non sans ironie tout simplement évoquer la formule credo quia absurdum; or il souligne au moins un fait dont les traducteurs et les interprètes sont familiers, celui de la non-compréhension qui, dans un contexte tout autre que celui des mondanités auxquelles pouvaient donner lieu l'Académie des sciences morales où Talleyrand siégeait, renvoie bel et bien au point de départ de ce que Schleiermacher appelle "herméneutique ». Cette dernière repose, comme il l'écrit, "sur le fait de la non-compréhension du discours ${ }^{2}$. Ce qui implique également, quant au travail requis par l'interprétation, "qu'on ne parvienne à la langue elle-même et à la connaissance des choses suprasensibles qu'à travers la compréhension des discours humains ${ }^{3}$. L'affirmation semble à première vue éminemment restrictive mais est, en réalité, la condition même à laquelle tout traducteur doit souscrire s'il ne veut pas donner très vite dans ce que le même Valéry fustigeait sous le terme de "superstition littéraire ": "J'appelle ainsi toutes croyances qui ont de commun l'oubli de la condition verbale de la littérature. ${ }^{4}$ C'est-à-dire oublier qu'un texte a d'abord tissé un réseau de connivences langagières vis-àvis desquelles il reste néanmoins libre des agencements que son auteur introduit et qu'il ne peut, la plupart du temps, signaler autrement que par des outils discursifs. D'un autre côté, il est rassurant de savoir qu'un texte qui nous semble incompréhensible, et le cas échéant le reste, ne le sera pas en raison de composantes totalement étrangères au monde du langage humain ${ }^{5}$. Mais il est indéniable qu'une

1. Valéry, $1957: 936$.

2. Schleiermacher, $1987: 73$.

3. Ibid.

4. Valéry, $1960: 568$.

5. Même lorsqu'il développe sa conception mystique du langage, Benjamin (2000 : I, 143165 - Sur le langage en général et sur le langage humain - et I, 244-262 - La Tâche du traducteur) ne suppose jamais qu'il faille être doté de capacités spéciales pour comprendre le sens de ce que serait tel mot dans l'ordre du «langage pur » ni que lui-même fût bénéficiaire d'une sorte d'illumination exceptionnelle lorsqu'il a développé sa conception de la traduction. 
situation herméneutique "normale » offre aux traducteurs et aux commentateurs certains éléments de comparaison qui permettent de mesurer un tant soit peu la manière dont l'auteur ou les auteurs d'un texte ont traité les matériaux discursifs qui leur étaient légués; sans ces éléments l'interprétation ne peut que se borner à être conjecturale. Faute de pouvoir réinstaller le texte dans une histoire, les traducteurs n'ont d'autre recours que d'interroger le texte lui-même dans sa structure en se passant de termes de comparaison puisés dans ce qu'on appelle le péritexte ou le contexte. C'est dans cette perspective qu'on peut comprendre le désarroi de certains traducteurs et commentateurs face à nombre de passages du Pentateuque dont la facture est d'emblée non seulement problématique, mais véritablement énigmatique, ce qui est le cas, notamment, de Gn 6,1-4. Comment reconstruire un original sans pouvoir apprécier la valeur respective des matériaux qu'on doit interpréter?

\section{Figures déroutantes}

6,1 Lorsque les hommes commencèrent d'être nombreux sur la face de la terre et que des filles leur furent nées,

6,2 les fils de Dieu trouvèrent que les filles des hommes leur convenaient et ils prirent pour femmes toutes celles qu'il leur plut.

6,3 Yahvé dit: "Mon esprit ne demeurera pas dans l'homme, puisqu'il est chair; sa vie ne sera que de cent vingt ans. »

6,4 Les Nephilim étaient sur la terre en ces jours-là (et aussi dans la suite) quand les fils de Dieu s'unissaient aux filles des hommes et qu'elles leur donnaient des enfants; ce sont les héros du temps jadis, ces hommes fameux.

Néanmoins, Benjamin déclare d'emblée suivre la Bible «dans son principe, en présupposant, avec elle le langage comme réalité dernière, inexplicable, mystique » (2000 : I, 152). Certes, Dieu parle et ses paroles sont créatrices, mais pas à chacun des six « jours » de la "Création ", puisque l'expression «Dieu dit » est employée deux fois, au sixième jour, non pour créer ou faire quelque chose, mais simplement pour enjoindre aux hommes de "croître et de se multiplier ", ainsi que pour leur offrir de commander à l'ensemble de la création, pour leur offrir également, à eux comme aux animaux, la nourriture provenant des végétaux et leurs fruits. La liaison entre « dire » et créer n'est donc pas systématique. D'autre part, l'acte divin de nommer s'applique moins à des choses qu'à des séparations (jour et nuit, eaux d'en haut et eaux d'en bas, humide et sec). Nommer tout le reste de la création incombe aux hommes, et le langage n'est jamais présenté dans le texte biblique comme une faculté spéciale : la puissance créatrice divine se manifeste également dans des actes qui n'ont rien à voir avec une expression langagière : séparer, placer, faire pousser, par exemple; ainsi le langage n'est-il ni un apanage divin ni une manière, pour l'homme, de s'élever vers une contemplation extatique du divin. En outre, nommer son vis-à-vis d'un nom commun $(\mathrm{Gn} 2,23)$ n'est toujours pas suffisant pour accéder véritablement à un langage réalisable dans une langue : il faut aussi accéder au nom propre (Gn 3,20), mais également prendre part à des situations illocutoires où il est impossible de parler à la place de l'interlocuteur. C'est d'ailleurs l'une des significations possibles de Gn 2,24: l'homme doit "quitter son père et sa mère ", sa tradition familière, pour s'attacher à sa femme : la désigner et la nommer ce n'est pas quitter le terrain du soi ; il faudrait encore faire droit pleinement à une raison étrangère, par nature imprévisible, du moins dans certaines de ses manifestations. 
Le désarroi s'exprime déjà dans la manière dont ces quatre versets sont situés par des traductions récentes dans la composition du texte; ainsi la Bible de Jérusalem leur donne-t-elle pour intertitre "Fils de Dieu et filles des hommes », tout en les excluant de ce qu'elle appelle "Chapitre II : Le Déluge » qui débute alors en $\mathrm{Gn}$ 6,5. Ces quatre versets ne peuvent plus alors être considérés comme un exposé des motifs qui ont conduit au déluge ${ }^{6}$. En note, les traducteurs ne justifient nullement le redécoupage qu'ils introduisent, mais indiquent : "Épisode difficile (de tradition yahviste). L'auteur sacré se réfère à une légende populaire sur les géants, en hébr. nephilim, qui seraient des Titans orientaux, nés de l'union entre des mortelles et des êtres célestes. " ${ }^{7}$ Comme le terme nephilim est repris dans le texte de la traduction sans autre explication, le lecteur ne peut évidemment pas apprécier sa valeur propre ni sa mise en regard avec les «fils de Dieu ». La traduction n'indique pas non plus nettement si nephilim est synonyme des « fils de Dieu » ou s'il est synonyme des « héros » engendrés par l'union desdits «fils de Dieu » avec les filles des hommes. L'introduction du terme de " géants », qu'on doit à la Septante, déclenche inévitablement une série connue d'associations avec la mythologie grecque (Titans, géants, unions des dieux avec les mortelles, demi-dieux). En effet, la version première du texte grec avait choisi angéloï pour revenir ensuite à un calque de l'hébreu beneï ha-Elohim, et gigantes pour traduire à la fois nephilim et gibborim ${ }^{8}$. Les choix de ces traductions ont le mérite d'installer une cohésion, mais cette dernière, trop immédiatement parlante pour nous, repose uniquement sur une représentation grecque des généalogies dont le présupposé est tout simplement que le monde des dieux se mêle à celui des mortels en créant des êtres exceptionnels. Un anachronisme s'installe insidieusement - masquant sans doute une autre source, mésopotamienne ou babylonienne, à laquelle, plus vraisemblablement, le texte emprunte les éléments dont il a besoin pour produire un effet de reconnaissance -, et se renforce par l'effet de « réalisme» second produit par notre propre réception de la mythologie, souvent comprise, en outre, de manière naïve comme un système de croyances effectives par rapport auquel les Grecs eussent été sans distance. Il ne suffit pas, en effet, d'évoquer des "Titans orientaux " ${ }^{9}$, car l'identification même

6. La Bible de Jérusalem (1973: 36 sq.)

7. Ibid., note $f$. Tout le reste de la note reconstruit, sans argumenter, ce qu'ont dû être les intentions de l' " auteur sacré " et admet que cet épisode évoque le "souvenir d'une race insolente de surhommes comme un exemple de la perversité croissante qui va motiver le déluge ». Autrement dit, la note contredit les choix de composition des intertitres et des titres, puisqu'elle rétablit cet épisode parmi les causes du déluge.

8. La Bible d'Alexandrie (1986: 125 sq.) Buber et Rosenzweig (1987: 22) distinguent entre Riesen (nephilim) et Helden (gibborim), en établissant une relation de synonymie entre les deux termes, sans toutefois rompre avec les connotations grecques introduites par la Septante.

9. La Bible de Jérusalem (1973: 36 sq., note f). Au demeurant, les Titans sont fils et filles du Ciel et de la Terre, et ne sont donc nullement engendrés par des unions entre "divins " et "mortelles »; en outre, ils s'attaquent à Ouranos. D’autre part, les Géants sont issus de la Terre-mère et du Tartare (Graves, 1996 : 42). 
hypothétique d'une source ne dit rien quant à la valeur propre de son importation dans le texte qui la cite ni sur celle de sa fonction à l'intérieur de ce même texte dont l' «hospitalité » n'est en rien passive. Cette tentation anachroniste, toute imprégnée d'évidences inaperçues qu'elle doit à son propre présent, risque de vouloir par trop goûter au «bonheur inestimable de contempler son propre visage, né d'hier, dans le miroir d'une antiquité aussi reculée » ${ }^{10}$.

D'autres traductions de la Bible sont loin de dissiper les interrogations : ainsi l'interprétation de Luther introduit-elle une variante en faisant des nephilim des «tyrans» et des gibborim les « hommes puissants»(Gewaltige), jouissant de renommée et engendrés par l'union des fils de Dieu et des filles des hommes. La liaison est indirectement établie entre nephilim et gibborim où la synonymie est maintenue avec une nuance explicative : les tyrans nés des fils de Dieu sont des hommes puissants, c'est-à-dire capables d'user de violence (Gewalt), et c'est très logiquement que Luther donne pour titre aux versets 6,1-8 «La méchanceté des hommes "; il indique ainsi une volonté de neutraliser autant que possible les connotations mythologiques (on le remarque également dans le choix qu'il fait d'utiliser Kinder (enfants) et non Söhne (fils) dans sa traduction de benë̈ ha-Elohim), et de considérer ces versets comme faisant bien partie de l'exposé des mobiles effectivement déclencheurs du déluge. La King James parle de sons of God et traduit nephilim par giants (une note indique la référence à $\mathrm{Nb} 13,33$ ), gibborim par mighty men, en établissant sans équivoque une synonymie entre les deux, les géants " devenant » des hommes puissants puisqu'ils sont engendrés par les fils de Dieu unis aux filles des hommes. La traduction de Segond donne pour titre général aux trois chapitres Gn 6,7 et 8 : "Corruption du genre humain - Le déluge », et traduit nephilim par " géants », gibborim par « héros ». Dhorme, dans sa traduction plus récente ${ }^{11}$, reprend finalement la même solution, étayée par des notes où se mêlent aperçus interprétatifs, renvois à des textes ultérieurs et indications étymologiques sans que le lecteur non averti puisse véritablement rien y distinguer, et conforte ainsi les inévitables déformations qui surgissent du prisme " mythologique ». Le problème n'est pas alors qu'on devrait comprendre ce texte à la lumière des Métamorphoses d'Ovide ${ }^{12}$, par exemple, mais qu'il faudrait pouvoir apprécier les différentes valeurs accordées à l'importation d'éléments mythologiques, et, de même, être en mesure d'en déduire le rôle qu'elle joue dans la composition du contexte.

10. Schelling, $1856: 29$.

11. Dhorme, $1956: 18$ sq.

12. Ovide, $1992: 47$ sq. et 51-57. 


\section{La référence mythologique}

Dans les Amours (III, 12, v. 41-42), Ovide affirme lui-même que "l'inspiration créatrice des poètes se donne libre cours et n'astreint pas ses paroles à la vérité historique ", ce que confirme l'épilogue des Métamorphoses où le poète, se vengeant de son exil, vante son œuvre qui lui vaudra une renommée plus durable que celle d'Auguste, et, surtout, affirme que la "vérité » propre aux «pressentiments des poètes » concerne l'art et non ce qui en est le matériau. Autrement dit, au tout début de l'ère commune, lorsque circulent à Rome des copies de cette œuvre, son auteur hérite d'un matériau qui n'est plus pour lui autre chose qu'un bloc de marbre, son art ayant démontré par d'autres livres qu'il pouvait s'exercer à partir d'autre chose que des récits mythologiques dont le contenu est désormais sans vie, c'est-à-dire n'est plus à même d'inspirer la réflexion. Tout autre est l'attitude d'un Hésiode qui ne récapitule pas, comme Ovide, les récits d'une tradition qui les a rabâchés, mais qui cherche, au contraire, à reconstruire une cohérence au sein de récits que son art ne traite pas comme un matériau parmi d'autres, car il envisage de les présenter de telle manière que la narration soit aussi la possibilité d'y déceler des vérités non pas simplement poétiques, mais touchant plus profondément l'ordre des choses. Les Muses, dans le poème de la Théogonie ${ }^{13}$, déclarent : "Nous savons dire des mensonges / qui ressemblent à du vrai. / Nous savons, quand nous le voulons, / dire à voix claire des vérités. » Un peu plus loin (vers 36-38), Hésiode souligne, à leur propos, qu' " elles disent ce qui est, / ce qui sera et ce qui fut jadis / et leurs voix s'accordent ». Huit siècles avant Ovide, la tradition mythologique n'est certes pas naïvement reçue ni ne fait l'objet d'une «croyance " dont la Théogonie témoignerait sans distance ; bien au contraire, le poète est déjà fort instruit dans la manière de présenter plusieurs strates combinées d'énonciation où démêler ce qui relève de la «fiction » et de la "vérité » est la tâche reconnue d'emblée incomber au lecteur. Mais surtout, c'est affirmer qu'aucun mode de communication de ce qui est vrai n'échappera à la "poésie » à laquelle doit obéir cette transmission; Schelling l'a tout de suite compris, même s'il croit disposer, par ailleurs, d'assurances plus profondes : «(...) au sein de la mythologie, une philosophie ne saurait être à l'œuvre qui aurait à aller chercher d'abord dans la poésie ses figures ; au contraire, cette philosophie était elle-même et par nature également poésie, et inversement. » ${ }^{14} \mathrm{La}$ lucidité et la maturité dont Hésiode fait ainsi preuve ne sauraient être refusées aux rédacteurs du texte biblique.

Mais une tentation peut alors surgir qui serait de conclure de la présence d'un « déluge » dans nombre de mythologies à une sorte d'universalité anthropologique de sa représentation: la réflexion s'orienterait ainsi vers une ethnopsychanalyse dont la tâche serait de démontrer la nécessité de pareille représentation

13. Hésiode, $2001: 34$ sq.

14. Schelling, 1856 : 52 (la pagination est celle de l'édition allemande qui figure dans la traduction française). 
dans ce qu'on supposerait être le cours obligé d'un développement de toute civilisation ${ }^{15}$. Aussi loin qu'on puisse poursuivre cette voie comparatiste, elle ne conduit qu'à dissoudre les textes et leurs différences dans une évolution où ces derniers ne seraient plus que les dépositaires de motions dont la logique propre pourrait fort bien se dispenser de toute sédimentation scripturaire. Les textes seraient ainsi commandés, très en amont de leur rédaction et à l'insu même de leurs auteurs, par les nécessités d'un sens éminemment antérieur et bien plus contraignant que celui qu'ils tentent de transmettre. L'inverse de cette position « holiste » mène également à une impasse : le différentialisme extrême voudrait que chaque culture fût à ce point spécifique et cloisonnée que les textes qui la singularisent n'obéiraient qu'à leur propre logique purement interne, chaque texte fonctionnant en quelque sorte comme un univers clos et autosuffisant, excluant tout autant la part active qu'eussent pu y prendre leurs auteurs. L'absurdité de cette position qui n'est en fait jamais concrètement adoptée - parce qu'impraticable - ne fait que révéler la fragilité de son avers universaliste ${ }^{16}$. L'essentiel est de souligner toute l'équivoque d'un jugement fondé sur la croyance en une histoire universelle ${ }^{17}$, car si tous les textes se ressemblent quand ils se font l'écho d'une étape nécessaire dans le développement général, comment expliquer que les auteurs aient la capacité d'innover en produisant des différences qu'on ne peut réduire à des variantes sans faire violence précisément aux textes mêmes ? Ainsi faut-il admettre que les auteurs et, bien entendu, les récepteurs immédiats du texte biblique ont eu connaissance non pas de la «mythologie » en général ni de la mythologie grecque, mais sans doute de textes et de mythologèmes assyro-babyloniens, et que l'apparition, dans le texte du Pentateuque, de termes et de séquences propres à cette source culturelle était reconnue et identifiée comme telle. Bref, les sources ne délivrent pas le sens du texte qui, précisément parce qu'il les exploite, les intègre d'abord en fonction de la valeur

15. C’est toute l'ambiguïté qui grève la lecture "historico-critique », malgré ses dehors rationalistes : "Une Aufklärung conséquente régresse en mythologie (...) lorsque, dans la foi en un donné ultime, elle interrompt la réflexion. »(Adorno, 1966 : 130) ; ici le donné ultime serait un donné historique dont la cohésion de sens serait secondaire (et souvent impossible à reconstituer) par rapport à ses significations contextuelles.

16. Dès le début de la formation moderne de «l'histoire universelle ", Vico affirme, dans sa Science nouvelle (1986 (1744) : \380) que "le déluge fut universel », et, de même, il érige en «postulat» $(\$ 193)$ ceci : "C'est Jupiter qui foudroie et terrasse les géants ; chaque nation païenne a eu son Jupiter ", postulat qui implique une autre affirmation nécessaire : dans le monde primitif, il faut distinguer "deux catégories d'hommes : les Hébreux qui forment le premier groupe et qui eurent une taille normale ; les géants fondateurs des nations païennes » (\$ 372). Du même coup, l'interprétation qu'il donne de Gn 6,4 (Livre II, chap. 3, "Du déluge universel et des géants ") admet que les fils de Noé gardèrent une taille normale ("élevés dans la crainte de Dieu et des pères ", $\mathbb{S} 370$ ) à cause de "l'horreur que leur inspirait la taille de certains hommes ".

17. Schelling, $1856: 61$ : «Il existe une parenté intime entre les mythologies des peuples les plus différents, et, au demeurant, les moins comparables »; à ses yeux, c'est un fait " que la mythologie soit un phénomène universel et, dans l'ensemble, partout identique ». 
requise par sa propre démarche. Dans les versets qui nous intéressent, et dans la présentation du déluge, on reconnaît sans peine la référence à l'épopée de Gilgamesh ${ }^{18}$. Les ressemblances sont multiples : certains éléments de diégèse, certains termes (kopher, "poix» ou "bitume de calfatage » est un calque; mabbul, le mot même qui désigne le déluge a peut-être une racine akkadienne, nabalu ou bubbulu), d'évidentes similitudes dans le détail de la construction de l' " arche ». Ce réseau de ressemblances a bien évidemment pour fonction principale de produire un effet de familiarité chez le lecteur qui ainsi " reconnaît » en les identifiant des portions d'une tradition reçue. Mais le texte biblique installe en même temps une série d'hapax : yadôn (v. 6,3 ; "séjourner »), tzohar (v. 6,16; « ouverture»), gopher (6,14; une certaine essence de bois), kopher (v. 6,14; une substance de calfatage); ou de mots assez rares qui apparaissent ici pour la première fois et ne sont repris que très rarement : mabbul, tebha (la boîte ou le coffre, traduit habituellement par "arche ", et qui n'apparaît ensuite qu'en Exode 2,3-5 pour désigner le couffin de roseaux qui permet à Moïse d'être sauvé des eaux), mais également beneï ha-Elohim (qui n'est repris que dans Job, c'està-dire dans un tout autre contexte scripturaire), nephilim qui semble être un terme à ce point étranger au lexique du Pentateuque qu'il a besoin d'être explicité par une sorte de quasi synonyme (gibborim, plus facile à comprendre : hommes puissants, voire violents) même s'il reparaît dans $\mathrm{Nb}$ 13,33. Autrement dit, à l'effet de reconnaissance se superpose l'effet inverse; et cette superposition d'orientations inverses semble justifier qu'un grand commentateur de la Genèse comme Cassuto déclare à propos de 6,14 que ce passage est «l'un des plus obscurs dans le Pentateuque ${ }^{19}$. En effet, Cassuto est très embarrassé par la présence d'éléments mythologiques dont il identifie d'ailleurs la provenance (les alliances entre dieux et mortels sont présentes dans les légendes cananéennes), mais qui, à ses yeux, contrecarre un dessein général du texte : «La Tora, au contraire, a l'intention de s'opposer aux légendes païennes et de réduire au minimum le contenu des anciennes traditions à propos des géants ${ }^{20}$.

\section{La lecture des philosophes}

On pourrait ainsi croire qu'un tel dessein trouverait systématiquement audience chez les commentateurs philosophes. Or l'unanimité est loin de régner, ne serait-ce que depuis deux siècles, dans la tradition philosophique lorsqu'elle fait écho aux versets de Gn 6,1-4. Il est remarquable, par exemple, qu'un Herder déploie une sorte de panégyrique poétique et plein d'une ardeur lyrique pour vanter, dans des termes qui confinent à l'érotisme dionysiaque, l'union des « fils de Dieu » avec les filles des hommes. Remarquable aussi, dans cet hymne au

18. L’Épopée de Gilgamesh, 1992 : 184-197.

19. Cassuto, $1961: 291$.

20. Ibid. : 300 . 
monde archaïque commandé par l'instinct, que Herder vante le chant de Lemekh, cet « esprit fort - héros, philosophe et poète ! ${ }^{21}$ qui « n'avait plus besoin de Dieu » ${ }^{22}$, comme si tout ce qui prépare le déluge n'était en somme que l'apogée d'un monde plus fort et moins fragile que celui des générations suivantes ${ }^{23}$. Schelling, lui, prend au sérieux ce passage "qui a toujours donné tant de mal aux interprètes ", en soulignant la présence de "situations mythologiques effectives » ${ }^{24}$, et qui, selon lui, est un témoignage historique d'une phase de transition entre "les atteintes toujours plus fortes du polythéisme » ${ }^{25}$ et la voie qui conduira finalement au monothéisme. Mais l'ensemble de l'épisode du déluge est « la simple ligne de partage entre deux époques, celle d'une race encore douée de force surhumaine, et celle d'une race devenue pleinement humaine et vouée à l'humanité, mais qui, par là même, s'abandonne au polythéisme " ${ }^{26}$. Schelling poursuit un raisonnement qui suppose une Révélation progressive et quasi dialectique du «vrai Dieu » qui, se manifestant à partir d'Enosh (Gn 4,26) en se différenciant du premier Dieu originel, ne devient véritable que grâce à l'intervention du polythéisme qui permet la distinction entre Dieu d'une lignée (celle d'Abraham) et Dieu de tel ou tel peuple - dérive dans laquelle sombre Noé lorsqu'il devient agriculteur (planteur de vigne), tandis qu'Abraham et une partie de sa descendance (Jacob le nomade et non pas Esaü qui, comme Noé, se sédentarise) restent fidèles au vrai Dieu que les premières générations vénéraient comme 《Dieu vrai implicite sans le différencier comme tel ${ }^{27}$. Mais la première génération n'était pas en mesure "d'accueillir cette Révélation ». La seconde génération a su appeler Dieu par son vrai nom, mais "la Révélation ne sera donnée que quand sera donnée également la première atteinte par le polythéisme ». Noé appartient alors à une génération où le polythéisme ne peut plus être entravé et «se répand sur le genre humain"; dans les générations suivantes, «le monothéisme au sens propre (...) et du même coup la Révélation sont conservés » 28 , pour se manifester pleinement avec Abraham, Isaac et Jacob. On comprend alors que la préoccupation téléologique prend le pas sur l'analyse stricte du texte, et que la logique d'une reconstruction de l'histoire universelle de la spiritualité l'emporte sur celle de la l'enchaînement des chapitres 4,5 et 6 où l'effort du texte

21. Herder, 1993 : 643 (Älteste Urkunde des Menschengeschlechts). Un peu avant (ibid. : 638), Herder écrit à propos du chant de Lemekh qu'on avait affaire au «poème le plus simple, le plus fort et le plus ancien sur la plus terrible et la plus magnifique invention, l'éclair de dieu dans la main de l'homme, l'épée ! ».

22. Ibid.: 657.

23. Dans une note (ibid. : 652), Herder considère que les cent vingt années de vie accordées à l'homme $(G n$ 6,3) rendent le texte douteux puisque cette sentence divine ne s'applique ni à Noé ni à ses fils, si bien qu'il vaudrait mieux "se débarrasser de toute cette fable ".

24. Schelling, $1856: 149$

25. Ibid. : 151 .

26. Ibid. : 152 .

27. Ibid. : 160 .

28. Ibid. 
porte sur une manière subtile, mais nette, de prendre ses distances avec des éléments mythologiques reconnaissables ${ }^{29}$. Un topos de la conception "mythologique » est l'énumération généalogique commandée par l'idée générale que les ancêtres sont dignes d'être imités et que les plus anciens jouissent d'une dignité supérieure. Le texte biblique reprend cette forme, mais la répète en introduisant quelques écarts significatifs : Adam, Caïn, Lemekh ne sont à l'évidence pas des figures prestigieuses, ils valent plutôt en fonction de certains de leurs descendants et non en raison de leur antériorité ; la vengeance interdite contre Caïn évacue, d'autre part, la possibilité d'une mort rachetant la faute et rétablissant dans son intégrité le cours des générations; pire, l'attitude de Lemekh apparaît comme une perversion de ce sens-là du « signe de Caïn » en suggérant que le meurtre qu'il vient de commettre restera sans châtiment ${ }^{30}$. Ainsi le texte introduit-il des effets de reconnaissance pour, en les répétant, installer une distance ironique. Et la même chose vaudrait, eu égard à l'interprétation de Schelling, pour l'interprétation de Gn 11,1-9, ainsi que pour le long cheminement de la promesse faite à Abraham de Gn 13 à 22. Ce que suggère le texte biblique, c'est précisément que cette promesse pourrait ne pas rencontrer les conditions qui lui sont nécessaires pour être réalisée : l'histoire humaine est présentée comme aléatoire et non comme la réalisation assurée d'un plan divin. Bien entendu, le texte sait par avance à quoi il doit aboutir ; mais la dramatisation de certains tournants décisifs a pour effet à la fois de faire valoir une probabilité corollaire de l'histoire, opposée à l'idée traditionnelle de destin, et, tout à la fois, de mettre en lumière des conditions de possibilité requises par l'établissement d'une «alliance » ou la réalisation d'une promesse spéciale, l'élection d'Israël.

Ainsi, même pris dans des acceptions difficilement conciliables, les « fils de Dieu » restent-ils d'énigmatiques figures quand bien même elles sont clairement référées à des sources mythologiques, car le statut de cette référence n'est pas explicité. En effet, beneï ha-Elohim semble être à la fois une sorte de "carré rond », du point de vue de la Tora, et une citation renvoyant à des récits vraisemblablement connus. Il ne suffit pas, pour comprendre ce syntagme, de noter l'effet de contraste beneï ha-Elohim / banot ha-adam, car l'union des "fils de Dieu » ou des « fils des dieux » avec les mortelles n'implique nullement qu'elle soit une cause du déluge effectivement raconté dans l'épopée de Gilgamesh (qui mentionne en outre un débat sur son éventuelle immortalité, donc sur la restriction de la durée de sa vie, qui pourrait trouver écho dans Gn 6,3); d'autre part, on ne saurait y voir expliciter l'idée d'une contamination par l'idolâtrie croissante dans la mesure où la première conséquence de pareille union est la réaction de

29. Sur les interprétations successives de Gn 6,1-4 par Fichte, Schelling et même Simone Weil (1950 : 223-236; 1966 : 229-246), on consultera Tilliette, 2001 : 35-48.

30. Un certain parallélisme dans l'onomastique des deux lignées, caïnite et sethite, suggère que ces générations iraient du pareil au même, et certainement pas du meilleur au médiocre ni l'inverse. 
Dieu vouant désormais les hommes à une vie plus courte, tandis que le rejet des idoles n'apparaît nulle part dans l'épisode du déluge.

L'interprétation du passage ne fait que gagner en complexité si l'on évoque des sources différentes, élohiste et yahviste, ce qui a été parfois le cas ${ }^{31}$, dans la mesure où restent tout aussi énigmatiques les raisons pour lesquelles les rédacteurs eussent eu la tentation d'entremêler ces sources, et de produire un texte composite sans dessein spécial. En effet, une autre opposition doit être soulignée entre Gn 6,2 et Gn 6,3, et à celle qui met en regard " fils des dieux » et " filles des hommes » vient s'ajouter celle entre ha-Elohim et Yahveh ( Alors Yahveh dit... »), suivie de la répétition beneï ha-Elohim $(6,4)$ et Yahveh $(6,5)$. L’hypothèse d'un entrelacement de sources disparates est alors on ne peut plus difficile à soutenir. En dépit des intérêts d'ordre téléologique qui commandent sa reconstruction d'une "philosophie de la mythologie », Schelling fait preuve d'une remarquable sagacité en refusant l'un des piliers de l'hypothèse "documentaire " : "On a cherché à fonder (...) l'hypothèse que la Genèse en particulier était composée à partir de deux sources : on appela la première la source élohiste, et la seconde la source yahviste. Or, on peut facilement se convaincre que, dans les narrations, les noms ne varient pas au hasard, mais qu'ils sont employés avec un discernement intentionnel, et que l'emploi de l'un ou de l'autre a son fondement dans la chose même et n'est pas déterminé par une circonstance simplement extérieure ou fortuite. » ${ }^{32}$ Bien avant Benno Jacob ${ }^{33}$, Schelling a donc l'intuition d'une intention particulière dans l'emploi des deux termes même s'il n'en définit pas les règles, comme le fera Cassuto de manière tout à fait cohérente du point de vue de l'interprétation juive traditionnelle ${ }^{34}$. Sans reprendre toute son argumentation, on peut noter que, de manière générale, "Yahveh ", le tétragramme, est employé pour exprimer le nom du Dieu d'Israël, tandis qu'Elohim a une fonction essentiellement appellative, qui peut tout aussi bien désigner des divinités païennes, ou lorsqu'il s'agit d'un syntagme lié ( "le Dieu d'Israël », le «Dieu de nos pères »), lorsqu'il est utilisé avec un pronom possessif, lorsqu'il a valeur adjectivale ( "vision de Dieu », pour vision divine). Ainsi Yahveh figure-t-il dans le texte lorsqu'on veut souligner un thème ou un mobile d'ordre éthique (un châtiment : 6,5-8; la droiture de Noah : 7,1); lorsqu'il est question de rituels proprement juifs $(7,5 ; 8,20-21)$; lorsqu'il s'agit d'exprimer une relation directe

31. L'annotation de la Bible de Jérusalem (de même, on l'a dit, son choix d'intertitres) et celle de l'Ancien Testament de Dhorme y ont recours, parmi bien d'autres exemples (tels Gunkel, 1901 : 50).

32. Schelling, $1856: 163$.

33. Jacob, 1913 : 93 sq. : "Le Pentateuque est un ouvrage qui a une unité. Son rédacteur l'a écrit à une époque riche de traditions historiques, en s'appuyant sur un vaste matériau littéraire, pour une part transmis oralement, pour une autre déjà travaillé par l'écrit, et il a fondu ces traditions, en en respectant les formes, dans une unité organique. L'élaboration des matériaux divers est le résultat d'un processus intensif, maîtrisé par la pensée de part en part. »

34. Cassuto, (1926) $1961: 19$ sq., 34 et 36 sq. 
entre Dieu et Noah $(7,16)$, ou encore pour marquer une différence dans l'avenir anticipé des deux fils de Noah (le fils de Ham étant maudit) quand leur père les bénit en utilisant le tétragramme pour Shem (appelé à être l'ancêtre d'Abram qui deviendra Abraham) et simplement Elohim pour Japhet. Le nom de Yahveh n'apparaît, employé seul pour la première fois, qu'en Gn 4,26, qu'une fois que le premier couple humain est assuré d'avoir des petits-enfants dans la lignée de Seth, dont le premier porte un nom, Enosh, dont le sens est quasi synonyme de celui de son grand-père ${ }^{35}$. Ainsi s'installe, plus profondément qu'au seul niveau sémantique, un réseau mêlant les axes sémiotique et sémantique, dont la dynamique s'amorce en Gn 4,26 (Enosh / Yahveh) et vient résonner entre Gn 5,32 (Shem, fils de Noah) et 6,9-10 (répétition du nom de Shem, fils de Noah, cette fois qualifié "d'homme juste dans ses générations "); on trouve en effet, encadrées par ces deux mentions des générations (justes) de Noah, les oppositions "fils d'Elohim » / "filles d'adam »; Elohim / Yahveh, puis la série nephilim / bneïha-Elohim / gibborim / anshé / ha-shem ${ }^{36}$, suivie par une quadruple mention de Yahveh $(6,5 ; 6,6 ; 6,7 ; 6,8)$.

\section{Générations}

Les trois fils de Noah mentionnés en 5,32 et répétés en 6,10, font l'objet d'une qualification particulière et indirecte en 6,9 (tzaddiq bedorothaw, "juste dans ses générations ") : ce sont eux, non pas chacun pris en lui-même, mais en tant qu'ils sont les engendrements de Noah, la génération qui s'annonce, différente de « cette génération-ci » $(7,1 ;$ bador ha-ze $)$. Autrement dit, ces engendrementslà, ces fils pris ensemble, constituent une génération qui pourra être sauvée du déluge parce qu'elle est "juste ", au contraire des engendrements entre les "fils des dieux » (ou « de Dieu » lato sensu) et les filles des hommes. Le passage du pluriel (ses générations) au singulier (cette génération) implique à la fois le rejet explicite de ce qui a pu être engendré par les "fils de Dieu» et les filles des hommes, par cette génération qui va subir le déluge (et qui englobe également Lemekh), et le jugement favorable porté sur les fils de Noah, dont la triplicité permet d'échapper au face à face qui vouait à un affrontement violent Caïn et Abel, sans recours à un tiers "yaphétique " ${ }^{37}$. Les noms de ces fils donnent une indication : Ham, le «bouillant, l'impulsif », rappelle évidemment Caïn ; Shem, le «nom », Abel, tandis que Japhet, «qu'il dilate » (« yéphet » : Gn 9,27) vient en tiers « dilatant » toute relation de face-à-face entre Ham et Shem, c'est-à-dire

35. Schelling va jusqu'à remarquer très finement qu'Elohim (et non Yahveh) est employé en Gn 17, à propos de la circoncision, car ce rituel n'est pas propre aux seuls Juifs, mais il n'en tire pas toutes les conséquences.

36. Littéralement: les « hommes du nom».

37. Ce qui suggère que parler requiert plus qu'un simple «vis-à-vis » $(G$ n 2,19) : « je » et «tu » doivent être complétés par un « il ». 
empêchant que ne se reproduise l'affrontement entre Caïn et Abel. Une indication indirecte est donnée si l'on suit un autre fil du texte depuis Gn 1,18-19, c'est-àdire depuis la formation du langage humain. La première tentative d'Adam qui consiste à nommer les animaux aboutit à une situation langagière qui reste en suspens : nommer des animaux ou des choses relève de la simple nomenclature, d'un lexique; mais un vocabulaire ne fait pas un langage, et la syntaxe reste alors absente tant qu'un "vis-à-vis ", un interlocuteur, fait défaut. C'est seulement lorsque la femme apparaît, donc d'abord dans la position d'interlocutrice, qu'est remplie la condition de possibilité du langage ; or, comme on peut aisément le constater, Adam et Ève ne se parlent pas - aucun dialogue entre eux ; Caïn s'adresse à son frère, mais sans doute le silence de ce dernier déclenche-t-il le meurtre dont il est victime pour partie responsable ; par la suite, aucun dialogue n'est rapporté ${ }^{38}$. En revanche, Lemekh s'adresse à ses femmes, mais, et sans qu'elles réagissent, c'est pour se vanter d'un meurtre dont il fait un chant. Aucun dialogue véritable n'est rapporté avant celui qu'on peut supposer avoir lieu entre les trois fils de Noah en Gn 9,22, même si les paroles qu'ils échangent ne sont pas mentionnées: Ham, profitant de la faiblesse physique de Noah entend " dire » à ses frères que leur père n'est qu'un géniteur, qu'il n'a donc rien d'autre à transmettre que du " corporel ». L'acte de Ham découvrant la nudité de son père n'aura pas d'autre conséquence violente que la malédiction de Noah vouant son fils à une condition serve, tandis que Shem est explicitement destiné à poursuivre la lignée qui vénérera Yahveh, et qu'à Japhet est offerte l'hospitalité "dans les tentes de Shem ». Les générations de Noah en font un homme juste parce qu'il est, à travers elles, porteur de l'avenir (Abraham descendant de Shem), et parce que ses trois fils ne répéteront pas le face-à-face mortel initial; même si la lignée de Ham sera mauvaise (Nimrod qualifié de gibbor ba'aretz, gibbor tzaïr li-pneï Yabveh, "violent sur la terre ", " chasseur puissant devant Yahveh "; qualification rappelant à dessein les gibborim), l'histoire peut se poursuivre sans que soit besoin de rappeler des conditions de possibilité dont le non-respect entraînerait sa perversion. La présence du mal dans l'histoire humaine n'est en rien une objection à son déroulement ni à la possibilité même d'une "alliance avec Dieu » (Gn 8,21 : "car le mal est depuis sa jeunesse présent dans l'imagination du cœur de l'homme »), ce qui, en outre, place sous un tout autre jour la question traditionnelle de la théodicée.

Quoi qu'il en soit, aux nephilim et aux gibborim nés de l'union des « fils des dieux » avec les filles des hommes s'oppose sans équivoque la justesse des engendrements de Noah. Mais en quoi les unions dont procèdent les nephilim sont-elles directement condamnables ? La première raison est obvie, et elle est appelée par Gn 4,19 : la polygamie est directement associée à une lignée caïnite,

38. En Gn 32, Jacob prend soin d'envoyer des messagers à son frère lorsqu'il revient vers Edom ; la médiation (et sans doute les présents !) évite le meurtre. Abel, «l'inspiré ", s'imagine pouvoir se passer de répondre à son frère. 
violente, dont la généalogie se clôt sur Tubal-Caïn, forgeron (et donc sans doute fabricant d'armes), et sa sœur Naama, tous deux enfants de Lemekh. L'indication que les «fils des dieux » choisissent des femmes renvoie à ce polygame ivre du crime qu'il a commis pour venger une blessure au point de chanter qu'il sera "vengé soixante-dix-sept fois », soit onze fois plus que Caïn, échappant donc à toute sanction normale et se plaçant hors toute loi, se faisant gloire de son impunité ; mais l'essentiel est ici que cette polygamie soit d'une nature toute différente puisque les filles des hommes ne seraient plus des femmes prises pour épouses, mais des êtres vivants naturels mis à disposition des caprices de ceux qui appartiendraient à un autre genre que l'humain, ou de ceux qui refuseraient ainsi de partager la même condition. L'union des «fils des dieux» avec les filles des hommes est ainsi une première fois placée sous le signe d'une certaine confusion qui va croissant chez nombre d'hommes. Mais une autre indication est donnée sous forme de citation directe : Gn 6,2 reprend Gn 3,6 ; ce que plusieurs commentateurs ont bien remarqué sans en tirer néanmoins toutes les conséquences ${ }^{39}$. Les «fils des dieux » commettent la même erreur qu'Ève, et, de même qu'alors le « châtiment » est indiqué dans le récit du bannissement du jardin d'Éden, ici, c'est le récit du déluge qui révèle ex post la gravité de la perversion dénoncée. "Voir que le fruit est bon » et vouloir le consommer ou "voir que les filles des hommes sont belles » et vouloir se les approprier, ces deux passages sont mis en parallèle pour dénoncer une même confusion : là, de la représentation et de la connaissance; ici, de la représentation et de la procréation, c'est-à-dire de la responsabilité dans le temps de l'histoire. Ève - mais tout autant Adam qui ne lui adresse pas la parole et obéit à la même motion - s'imagine que sa représentation, gagée sur la seule impression sensible de l'agréable, vaut pour jugement, et qui croit que la connaissance est du même coup chose consommable - pire, qui veut être "comme les dieux » en matière de «connaissance du bien et du mal ", sans comprendre la différence essentielle entre savoir quelque chose sur le bien et le mal et être le bien, entre posséder un savoir et agir moralement, entre s'approprier magiquement (manger) un tel savoir, sur la seule base de la vision empirique, et comprendre l'ordre divin qui, lui, relève de l'écoute et du « déchiffrement ", c'est-à-dire de la compréhension non contemplative qui suppose l'usage discursif du langage et la discussion (ce à quoi le texte biblique invite des lecteurs). Ève, donc, et Adam ouvrent la voie à des dérives (meurtre, impunité du crime) où ne manqueront pas de donner, durant dix générations, leurs « descendants ». La succession des générations, on l'a dit, mime un topos mythologique et produit un effet de reconnaissance; mais le nombre arrêté de ces générations indique qu'elles sont fictives : ce qui importe alors est le parallèle entre ces dix premières générations et les dix autres qui conduisent de Noah à Abraham (peutêtre s'agit-il d'une indication quant à la maturation nécessaire pour qu'une « catastrophe» finisse par s'intégrer à une histoire collective). Les « fils des

39. Westermann, $1974: 495$; et surtout Wénin, $2007: 183$ sq. 
dieux " portent cette confusion à son comble; et d'abord parce qu'ils ne sont pas effectivement ce qu'ils prétendent être ni ne sauraient disposer des filles des hommes à leur guise, comme s'il s'agissait seulement de les choisir en fonction du fugitif bénéfice narcissique offert par leur «beauté ». C'est cette perversionlà que visent les versets $6,11-12$ où la violence qui y préside et surtout en résulte est dénoncée.

\section{Enfants de la confusion}

Toute une lignée d'exégètes, depuis Philon (De gigantibus, II, 358), a pris au sérieux l'idée que les «fils de Dieu » pourraient être des anges, mais, souvent, pour des raisons qui confortaient leur volonté de montrer à quel point le texte biblique était grevée de mythologèmes. C'est, bien entendu, le cas d'un des grands représentants, au début $\mathrm{du} \mathrm{XX}^{\mathrm{e}}$ siècle, de la lecture historico-critique : Hermann Gunkel n'hésite pas à donner pour titre à Gn 6,1-4 «Les mariages des anges » (Gerhard von Rad lui emboîte le pas ${ }^{40}$ ), et même s'il est obligé de reconnaître que l'expression est extérieure à la religion de Yahveh, il souligne que «Gn 6 est si éminemment mythologique qu'il a suscité des soupçons chez de nombreux interprètes d'époques ancienne et récente, et que ce passage a bien dû se soumettre à d'inévitables dérives interprétatives " ${ }^{41}$. Plus surprenant se révèle le commentaire de Jan Alberto Soggin qui refuse de voir dans les " fils des dieux » autre chose que des êtres divins, tout en admettant qu'ainsi " reste à vrai dire sans solution le problème suivant : pour quelle raison une humanité innocente aurait à subir les conséquences des péchés de ces êtres divins ? " Mais l'échappatoire est là, il suffit d'accuser le texte de n'être pas à la hauteur de nos attentes : "Il semble que ce soit la manière dont pense et agit le mythe. " ${ }^{42}$ Benno Jacob, l'adversaire précoce de l'hypothèse documentaire, rappelle une évidence : «Il faut d'abord partir du fait que le passage est censé servir le contexte où il se trouve. Parler d'une importation étrangère, d'un fragment égaré, etc., n'est qu'avouer qu'on n'a pas encore compris ce contexte. » Et il indique que Gn 6,1-4 introduit et justifie le récit du déluge qui doit frapper les habitants de la terre, si bien qu'il faut chercher les coupables parmi eux et nulle part ailleurs. Les « fils des dieux " sont donc "à tous égards des hommes " ${ }^{43}$. Que l'expression soit frappante n'est en rien surprenant puisqu'il s'agit de dramatiser les dérives des dix générations précédentes que dix générations ${ }^{44}$, de Noah à Abraham, auront

40. Von Rad, $1973: 113$.

41. Gunkel, $1901: 50$ sq.

42. Soggin, $1997: 120$.

43. Jacob, 2000 [1934] : 172 (cf. pp. 170-171, sa longue réfutation des interprétations contradictoires qui ont été données : les « fils des dieux » sont des anges ou ce sont, au contraire, des hommes pieux issus de la lignée de Seth).

44. L'effet de symétrie est évidemment voulu (et ne reste pas sans échos : dix paroles créent le monde; dix commandements constituent la Révélation au Sinai), mais, surtout, cet effet de 
à corriger, non sans connaître elles-mêmes des régressions (le mal fait partie de l'histoire humaine ${ }^{45}$ ), comme le montre de manière exemplaire l'épisode de Babel (Gn 11,1-9) qui vient interrompre, dans la disposition même du texte, l'énumération des descendants de Shem. Le texte recourt ainsi à une expression dont la connotation sémiotique renvoie à l'univers mythologique, connu mais tenu à distance par l'effet de citation, et la connotation sémantique à une impossibilité logique : le sémantème ainsi formé souligne bien l'impasse où se trouve cette humanité fourvoyée. Et la descendance de ces hommes qui se prennent pour des fils de Dieu ou font croire qu'ils sont tels ne saurait être le moins du monde vantée par le contexte imputant à leurs errements le déclenchement du déluge, mais d'abord la restriction de la durée d'une vie humaine dans la mesure où la filiation divine implique une volonté de nier la finitude même : le fait que l'adam est composite, qu'il bénéficie du souffle divin, certes, mais reste prisonnier de la terre, elle-même présente dans son nom. On comprend ainsi la raison d'être de l'hapax yadôn: la question de l'union de l'âme et du corps n'est pas un problème que les hommes ont à se poser réellement pour savoir comment s'orienter dans la vie morale et politique. Le mystère que constitue la présence en nous de ce souffle divin doit rester une énigme (ou doit être soustrait à l'examen de tout un chacun au profit de la réflexion de ceux qui seraient spécialement en mesure de le faire et disposeraient d'une légitimité particulière). Mais l'essentiel n'est pas le sens plus ou moins « technique » du terme qu'il est sans doute impossible à reconstituer ; ce qui importe, c'est la variation sur la durée et la réaffirmation qu'il faut bannir tout rêve d'éternité chez les hommes - le texte poursuit Gn 3,22 -, en réservant «ce qui dure éternellement » à la sphère éthique. Encore une fois, la vie humaine se déroule dans le registre ouvert par toledot, et le désir d'accéder à olam reste interdit ${ }^{46}$.

Ceux qui sont tentés d'échapper au nom d'adam en s'affublant d'une désignation qui, précisément, efface la finitude, peuvent donner à entendre que leur vie se prolongera indéfiniment, grâce au rajeunissement fictif, mais à leurs yeux concret, que leur procure la séduction réitérée de femmes belles. Cette illusoire " éternité » s'achève d'emblée dans leur progéniture, les nephilim dont la connotation sémiotique dit bien ce qu'ils sont : des déchus ${ }^{47}$. Ils ne sont pas «tombés

symétrie indique bien quel rôle de charnière incombe au déluge dans la représentation ainsi proposée d'une "histoire " éthique. La numération exacte des généalogies produit un effet ironique par rapport à tout "réalisme ", et oriente vers le symbolique en s'opposant à la fiction sans règle des lignées mythologiques : l'histoire effective est d'emblée référée par le texte biblique à ce qui doit en être le cadre nomothétique.

45. La lâcheté d'Abram durant son voyage en Égypte (Gn 12,11-19 où réapparaît le syntagme "ils virent qu'elle [Sarai] était belle ») en donne un témoignage plus poignant encore.

46. Abraham invoque le nom de Dieu en utilisant la désignation el olam en Gn 21, 33, pour parachever un accord simplement politique avec Abimelekh, et, tout de suite après, il est soumis à l'épreuve d'avoir à sacrifier Isaac : il vient de compromettre son avenir.

47. C'est ce que suggère du moins l'hypothèse étymologique qui rattache ce terme à la racine nâphal, " tomber». 
du ciel » ni ne sont des "géants ", mais bien des décadents. L'explicitation de la connotation sémantique offerte par gibborim ne doit pas non plus induire en erreur. Certes, il s'agit d'êtres puissants, mais cette puissance est d'abord celle d'une violence transgressive, car ils sont bien les enfants de la confusion. En outre, comment donner une inflexion positive à la manière dont ils sont qualifiés anshêi ha-shem? Comment voir en eux des "hommes fameux ", sauf à ne prendre en compte que la référence mythologique encore une fois citée ? Comment ne pas entendre ha-shem dans une acception tout simplement antiphrastique dans la suite logique de l'emploi de beneï ha-Elohim ? Les "géants » n'ont pas inquiété la terre d'après le déluge, même si Nimrod peut à juste titre passer pour un esprit hanté par des transgressions antérieures. Dans d'autres contextes, gibborim peut effectivement désigner des hommes de taille exceptionnelle, d'autant plus qu'il en est fait mention (sous le terme nephilim) dans le compte rendu des éclaireurs envoyés par Moïse en Canaan, et qui ne manquent pas alors de dramatiser les conditions de la conquête ( $\mathrm{Nb} \mathrm{13,33} \mathrm{et} \mathrm{Deut} \mathrm{2,21).} \mathrm{Ces}$ "géants » n'ont pas plus d'existence effective que leurs pères : ce sont des décadents violents, des hommes de belle réputation ! On se souvient de l'opposition Elohim / Yahveh encadrée par les deux mentions des fils de Noah, dont Shem : ha-shem entre ainsi dans une opposition qui sera, d'ailleurs, reprise lors de l'épisode de Babel où ceux qui veulent se soustraire à la loi de l'humanité - se disperser, former des peuples différents et parler des langues différentes - songent à « se faire un nom » (Gn 11,4), verset immédiatement suivi par "Yahveh descendit... » et le retour à la situation historique effective, c'est-à-dire la confusion de leur langage et leur dispersion. Ces gibborim, ces guerriers ${ }^{48}$, eux aussi ont voulu se faire un nom et se doter d'une réputation; elle ne peut être, au regard du texte, que répréhensible même s'il reconnaît en même temps que de tels hommes puissent passer pour fameux aux yeux de ceux et de celles qu'ils ont dupés, fascinés sans doute par leur pouvoir transgressif, a fortiori s'ils parvenaient à se faire passer pour des fils de Dieu (on notera ici l'absence de filles et de femmes).

\section{En l'absence de graphèmes}

Le texte biblique ignore nos graphèmes, tels le point d'exclamation ou les guillemets; c'est donc par d'autres moyens, sans doute plus subtils et plus complexes à mettre en œuvre, qu'il tisse un réseau de sens en jouant sur une distribution inversée de ce dernier : l'axe sémiotique déjouant sans cesse les prétentions ou les provocations de l'axe sémantique. L'ironie du texte à l'égard de ce qu'il installe se déploie néanmoins et parvient à se faire entendre en mobilisant toutes les ressources de langage dont il dispose pour à la fois maintenir la cohésion

48. Cf. l'interprétation de ce terme dans une perspective historico-sociologique, et à partir de textes postérieurs à la Genèse, chez Max Weber $(1970,38,42)$ qui voit en eux des propriétaires fonciers capables de prendre les armes. 
d'un récit fabuleux - adressé au plus grand nombre - et, dans cette cohésion même ou dans ce qui vient l'interrompre, susciter l'intérêt - de ceux qui pourront se passer du récit - pour une cohérence plus profonde qui, elle, relève de la réflexion éthique et politique sur les conditions initiales de possibilité des communautés humaines.

Marc DE LAUNAY

CNRS, Archives Husserl de Paris

marc.de.launay@ens.fr

\section{Bibliographie}

Adorno Theodor, 1966, Negative Dialektik, Frankfurt am Main, Suhrkamp [Dialectique négative, 1978, Paris, Payot, trad. fr. par le Groupe de traduction du Collège de philosophie].

Benjamin Walter, 2000, Euvres, 3 vol., Paris, Gallimard, "Folio-essais » (trad. M. de Gandillac, rev. par R. Rochlitz et P. Rusch).

Bible d'Alexandrie (La), 1986, Paris, Éditions du Cerf, (Marguerite Harl, éd.).

Bible de Jérusalem (La), 1973, Paris, Éditions du Cerf.

Bottero Jean, 1992, L’Épopée de Gilgamesh, 1992, Paris, Gallimard, coll. «L'aube des peuples".

Buber Martin, Rosenzweig Franz, 1961, A Commentary of the Book of Genesis, Part 1,

From Adam to Noah, Jérusalem, The Magnes Press-The Hebrew University.

-, 1987, Die Schrift, Heidelberg, Lambert \& Schneider.

Cassuto Umberto, [1926] 1961, The Documentary Hypothesis, Jérusalem, Magnes Press-

The Hebrew University.

Dhorme Édouard, 1956, La Bible. L’Ancien Testament, Paris, Gallimard, coll. «La Pléiade ".

Graves Robert, 1996, Les mythes grecs, Paris, Hachette-Pluriel, (trad. fr. M. Hafez). [Greek Myths, 1958].

GunKel Hermann, 1901 [éd. rev. en 1910], Genesis. Handkommentar zum Alten Testament, Göttingen, Vandenhoeck.

Herder Johann, 1993, Werke V, Schriften zum Alten Testament. Älteste Urkunde des Menschengeschlechts, Frankfurt am Main, Deutscher Klassiker Verlag.

HésIode, 2001, Théogonie et autres poèmes, Paris, Gallimard, «Folio-classique » (trad. fr. J.-L. Backès).

Jасов Benno, 1913, Die Thora Moses, Frankfurt am Main, J. Kauffmann, coll.

"Volksschriften über die jüdische Religion».

-, [1934] 2000, Das erste Buch der Tora. Genesis, Stuttgart, Calwer Verlag.

Ovide, 1992, Les Métamorphoses, Paris, Gallimard, «Folio-classique »(trad. fr. G. Lafaye).

RAD Gerhard von, 1973, Genesis. A Commentary, Philadelphie, The Westminster Press.

[Das erste Buch Moses. Genesis, 1972].

SCHELling Friedrich, 1856, Einleitung in die Philosophie der Mythologie, Stuttgart, Cotta.

[Introduction à la philosophie de la mythologie, Paris, Gallimard, 1998, coll. « Bibliothèque de Philosophie ", trad. fr. sous la dir. de J.-F. Marquet et J.-F. Courtine]. 
58 - ArChives de SCIENCES Sociales des Religions

SCHLeiermacher Friedrich, 1987, Herméneutique, Paris, Éditions du Cerf, coll. "Passages » (trad. fr. Ch. Berner). [Hermeneutik und Kritik, 1838].

SogGIN Jan A., 1997, Das Buch Genesis. Kommentar, Darmstadt, Wissenschaftliche Buchgesellschaft.

Tilliette Xavier, 2001, Les Philosophes lisent la Bible, Paris, Éditions du Cerf, coll. "Philosophie et Théologie ».

VAlÉRY Paul, 1957, Euvres I, Paris, Gallimard, coll. «La Pléiade ».

-, 1960, Euvres II, Paris, Gallimard, coll. « La Pléiade ».

VIco Giambattista, 1986, Principes d'une science nouvelle relative à la nature commune des nations, Paris, Nagel. [Scienza nuova, 1744].

Weber Max, 1970, Le judaïsme antique, Paris, Plon (trad. fr. F. Raphaël). [Gesammelte Aufsätze zur Religionssoziologie, vol. III, Tübingen, 1920].

WeIl Simone, [1940-1942] 1950, Attente de Dieu, Paris, La Colombe [Paris, Fayard, 1966].

WéNIN André, 2007, D’Adam à Abraham ou les errances de l'humain, Paris, Éditions du Cerf, coll. «Lire la Bible ».

Westermann Claus, 1974, Genesis, Neukirchener-Vluyn, Neukirchener Verlag.

\section{Résumé}

Le texte formé par les versets 1 à 4 du chapitre 6 de la Genèse est généralement considéré comme énigmatique en raison des expressions qui y figurent: "fils de Dieu ", nephilim et gibborim. Son rapport au récit du déluge est souvent discuté en liaison avec les sources mythologiques qui s'y manifestent. La plupart des traductions, dès la Septante, comme les interprétations issues de la tradition philosophique, renvoient le lecteur à un réseau de connotations mythologiques très reconnaissable qui passe à côté du travail subtil effectué par le texte, tant en amont qu'en aval de ce passage, et qui seul permet d'en comprendre les intentions véritables. C'est à reconstruire ce réseau que s'attache la présente étude.

Mots-clés : Déluge, fils des dieux, géants, mythologèmes, sémantèmes, traduction.

\section{Abstract}

Genesis 6, 1-4 is usually regarded as an enigmatic text because of the expressions it contains: "son of God", nephilim and gibborim. Its relationship to the tale of the flood is often discussed in connection with the mythological sources it reveals. Most of the translations beginning with the Septuagint, like the interpretations derived from philosophical tradition, take the reader back to a very recognizable network of mythological connotations which misses a subtle working carried out by the text, both before and after this passage, and which is the only thing that makes it possible to understand its true intentions. The present study is devoted to the reconstruction of this network.

Key words: the Flood, sons of gods, giants, mythologemes, semantemes, translation. 


\section{Resumen}

El texto formado por los versículos 1 a 4 del capitulo 6 del Génesis es generalmente considerado como enigmático debido a las expresiones que en él figuran: "bijos de Dios”, nephilim y gibborim. Su conexión con el relato del diluvio es a menudo discutido en relación con las fuentes mitológicas que se manifiestan. La mayoría de las traducciones, desde la Septante, como las interpretaciones salidas de la tradición filosófica, remiten al lector a una red de connotaciones mitológicas muy reconocibles que dejan de lado del trabajo sutil efectuado por el texto, tanto antes como después de este pasaje, que es lo único que permite comprender las intenciones verdaderas. El presente estudio se propone reconstruir esta red. (trad. Véronica Giménez Béliveau) Palabras clave: Diluvio, hijos de Dios, gigantes, mitologemas, semantemas, traducción. 